\title{
Chronic pain after spinal cord injury: a survey of practice in spinal injury units in the USA
}

\author{
AJ Ravenscroft ${ }^{*}, 1$ \\ ${ }^{1}$ Department of Spinal Injuries, Pinderfields General Hospital, Wakefield, Yorkshire, UK
}

\begin{abstract}
Objective: To determine the current practice regarding assessment and management of patients with chronic pain after spinal cord injury (SCI) in the United States of America (USA).

Methods: A postal questionnaire sent to the medical directors of 12 spinal injury units in the USA.

Results: A response was received from eight of the 12 units. Chronic pain was considered a significant problem amongst patients with SCI. There was inconsistency of opinion regarding prevalence estimates, investigation and management of chronic pain after SCI; but classification systems for pain were remarkably similar amongst units. Most felt that there was a need for further information, although only one unit said it was presently conducting research into the subject.

Conclusion: Our survey has demonstrated the uncertainty that exists amongst USA specialists dealing with pain after SCI, and strengthens the case for more research into the subject with a view to developing guidelines for care.
\end{abstract}

Spinal Cord (2000) 38, 658-660

Keywords: pain; spinal cord injury; dyaesthesia; central pain

\section{Introduction}

Our recent postal survey of UK spinal injury units aimed to determine current practice regarding assessment and management of patients with chronic pain after SCI. ${ }^{1}$ The results showed inconsistency of opinion amongst UK specialists regarding prevalence, aetiology, classification and effective management of chronic pain after SCI, and highlighted the need for more information. We have now performed a survey of 12 spinal injury units in the USA using an identical questionnaire. $^{1}$

\section{Results}

Responses were received from eight of the 12 units. Estimated prevalence of chronic pain after SCI ranged from $5 \%$ to $70 \%$ between respondents (mean $46 \%$ ). Six out of the eight units recognised chronic pain as a major problem amongst their patients, resulting in disrupted rehabilitation and depression. The most common factors felt to be associated with the development of chronic pain after SCI were incomplete injury (5/8 respondents) and thoracic injury (4/8). Two respondents observed that patients with SCI secondary to gunshot wounds were very likely to develop chronic pain.

*Correspondence: AJ Ravenscroft, Department of Anaesthetics, City Hospital, Nottingham, UK
Each unit described their classification system for pain after SCI using a list of categories into which they would place a patients pain type (Table 1). Five out of eight units felt it would be useful to have an internationally agreed taxonomy. Only two units had protocols for the investigation of chronic pain in their patients. Six out of the eight respondents felt it would be useful to have guidelines for the investigation and management of chronic pain in their patients. Management of pain involved several medical specialities including consultant psychologist (seven out of eight units), consultant in spinal injuries (5/8), consultant in pain management (5/8), physiotherapist $(4 / 8)$ and surgeon $(4 / 8)$.

Treatments tried were as follows: antidepressants (7/8), non-steroidal anti-inflammatory drugs (NSAIDs) $(6 / 8)$, anticonvulsants $(5 / 8)$, oral opiates $(1 / 8)$, transcutaneous nerve stimulation (TENS) (3/8), acupuncture (2/8), hypnotherapy (2/8), dorsal root entry zone (DREZ) lesioning $(2 / 8)$, intrathecal infusions of morphine $(3 / 8)$ or baclofen $(2 / 8)$. No units used oral ketamine, or the interventions of cordectomy or anterolateral cordotomy for pain control. Five respondents thought that starting some form of treatment immediately after injury might have an influence on the number of patients who develop chronic pain. The only suggestion for such a treatment was the anticonvulsant gabapentin. 
Table 1

\begin{tabular}{lllll}
\hline Unit & Category 1 & Category 2 & Category 3 & Category 4 \\
\hline 1 & Neuropathic & & Mechanical & Complex \\
2 & Central deafferentation & Segmental & Musculoskeletal & $\begin{array}{l}\text { Visceral } \\
\text { Psychogenic }\end{array}$ \\
3 & Neurogenic/deafferentation & Neurogenic zone of injury & Mechanical/orthopaedic & Referred \\
4 & Neurogenic & Musculoskeletal & Mixed \\
5 & Neurogenic & Phantom & Musculoskeletal & Post operative \\
6 & Neurogenic & Radicular & Nociceptive & \\
7 & Neurogenic/deafferentation & Central/Spinal vs peripheral & Nociceptive & Secondary gain \\
8 & Neurological & & & \\
\hline
\end{tabular}

This table shows the symptoms described by USA spinal injury units for the classification of pain after spinal cord injury

Seven out of the eight units felt that there was a need for more information on chronic pain after SCI, but only one said they were presently conducting research on the subject.

\section{Discussion}

This survey demonstrates similar inconsistencies regarding prevalence estimates, investigation and management of chronic pain after SCI amongst USA specialists as were seen in the UK survey. Interestingly, classification of pain is more consistent than in the UK, with most units describing pain on the basis of proposed mechanism or site of origin of pain. In contrast, UK specialists used many different systems including site, type and pain severity and consequences, as well as the above. A possible reason for the difference is that all of the American units belong to a federally designated and funded group of facilities called the 'Model System for Spinal Cord Injury', which requires them to meet rigorous standards of care determined by the US government.

Despite this, there were marked differences between the USA units regarding investigation and management of pain, and most units perceived a need for guidelines to improve this. Specialists involved in management were the same as in the UK, with a clinical psychologist involved in all units except one, but in USA units the consultant in spinal injuries was only involved in 5/8 units and the physiotherapist in $4 / 8$, whereas all of the UK units involved these specialities (9/9). A consultant in pain management was more frequently involved in the USA (5/8 cf. $4 / 9)$.

Treatments were broadly similar in the two countries, with widespread use of antidepressants, NSAIDS, and anticonvulsants, but UK units reported success using TENS (7/9) and oral opiates (5/9) for the relief of chronic pain, whilst only $3 / 8$ USA units had used TENS and only one had used opiates for this purpose. DREZ lesions were also less frequently tried in the USA $(2 / 8 \mathrm{cf}$. 6/9).

To interpret which treatments were considered effective for which types of pain, requires more accurate definition of pain type, and was considered beyond the scope of this survey.

\section{Conclusion}

The conclusions that can be drawn from this survey are limited by the small number of units involved and the selection of members of the 'Model system for spinal cord injury', which may not reflect practice in the remainder of USA units. In addition, the survey aimed to provide only an overview of pain management in these units, and further investigation is required to obtain more detailed information. This would include analysis of management protocols in use and their outcome data; specific definitions for pain types used in the classification systems, and an attempt at correlating common treatments to pain type and aetiology.

However, the results suggest that the uncertainty and inconsistencies regarding chronic pain after SCI identified in the UK extend to the USA, and strengthens the case for more research into the subject with a view to developing guidelines for care. It is interesting to note that although approximately $50 \%$ to $60 \%$ of the activity of the model system centres in the USA is devoted to clinical research, and seven out of eight units felt that there was a need for more information on chronic pain after SCI, only one unit said it was presently conducting research on the subject.

Concerning the management of pain after SCI, it is important to emphasise that like other chronic pain syndromes, it requires a multidisciplinary team approach. For many patients investigations will not identify a remedial cause for pain, and interventions will fail to provide relief. Management must therefore focus on improving function, quality of life and the ability of the individual to cope with pain.

Adequate time and explanation are essential in order to provide reassurance and overcome fears that chronic pain is a sign of harm and requires rest. A programme of physical retraining can improve function through paced activity, simple regular exercise, goal setting and improved sleep patterns. The impact of chronic pain on the sufferer should be 
considered and may reveal depression, anxiety or loss of self-esteem. Psychosocial assessment may also identify negative attitudes and beliefs, or inappropriate illness behaviour which may be contributing to distress and disability.

This multidimensional approach concentrates on the biopsychosocial model rather than the biomedical model used for acute pain problems, and has the aim of rehabilitating the patient back into a meaningful role in society.

\section{References}

1 Ravenscroft AJ, Ahmed YS, Burnside IG. Chronic pain after spinal cord injury. A survey of practice in UK spinal injury units. Spinal Cord 1999; 37: 25-28. 